\title{
Probing the revamped A4 symmetry at long-baseline neutrino experiments
}

\section{Sabya Sachi Chatterjee}

Institute of Physics, Sachivalaya Marg, Sainik School Post, Bhubaneswar 751005, India.

E-mail: sabya@iopb.res.in

\section{Mehedi Masud ${ }^{* \dagger}$}

AHEP Group, Institut de Física Corpuscular - C.S.I.C./Universitat de València, Parc Cientific de Paterna.

C/Catedratico José Beltrán, 2 E-46980 Paterna (València) - SPAIN.

E-mail: masudeific.uv.es

\section{Pedro Pasquini}

Instituto de Física Gleb Wataghin - UNICAMP, 13083-859, Campinas SP, Brazil.

E-mail: pasquinieifi.unicamp.br

\section{J.W.F. Valle}

AHEP Group, Institut de Física Corpuscular - C.S.I.C./Universitat de València, Parc Cientific de Paterna.

C/Catedratico José Beltrán, 2 E-46980 Paterna (València) - SPAIN.

E-mail: valledific.uv.es, URL: http://astroparticles.es

\begin{abstract}
In this work, we focus on the ability of the present and future long baseline neutrino experiments to probe the A4 symmetry based Babu-Ma-Valle model, revamped by including a flavon field. We perform realistic simulations of the experiments DUNE and T2HK and illustrate their potential to probe the model by considering two different set of best fit values. In particular, we show that the $3 \sigma$ allowed spaces in the plane of $\mathrm{CP}$ phase and the atmospheric angle, point to a lower octant for maximal CP violation ( $\delta_{c p} \sim-\pi / 2$ ), while the scenario of $\mathrm{CP}$ conservation points to a higher octant of the atmospheric angle. We also analyze how the capability of reconstructing the value of the CP phase and the atmospheric angle get significantly modified within the model. Finally, we perform a comparative study for DUNE and T2HK in order to probe the model by doing a full parameter scan (fit-independent) of the $\mathrm{CP}$ phase and the atmospheric angle.
\end{abstract}

The 19th International Workshop on Neutrinos from Accelerators-NUFACT2017

25-30 September, 2017

Uppsala University, Uppsala, Sweden

\footnotetext{
* Speaker.

${ }^{\dagger}$ Corresponding author
} 


\section{Introduction}

The observed flavor structure of quarks and leptons is unlikely to be an accident. Specially puzzling are the neutrino oscillation parameters [1], featuring two large angles with no counterpart in the quark sector [2], as well as a smaller mixing parameter measured at reactors, and which lies suspiciously close in magnitude to the Cabbibo angle [3, 4]. While the Standard Model gives an incredibly good description of "vertical" or intrafamily gauge interactions, it gives no guidance concerning "horizontal" interfamily interactions. A reasonable attempt to shed light on the pattern of fermion masses and mixings is the idea of flavor symmetry [5, 6, 7].

In this paper we consider a model suggested in [8], i.e. the simplest flavon generalization of the $A_{4}$-symmetry-based BMV model [9].

We focus on the capability of future experiments DUNE [10] and T2HK [11] to test the predictions of this model given the current measurements of the oscillation parameters.

\section{Theoretical preliminaries}

The model is a minimal extension of the BMV model [9], which assembles the $S U(2)_{L}$ doublet fermions into an $A_{4}$ triplet within a supersymmetric framework. It requires the existence of extra heavy fermions and three scalars $\chi_{i}, i=1,2,3$, all of them belonging to $A_{4}$ triplets representation and coupled through standard Yukawa interactions. Both standard Higgs fields $H_{i}$ and the three new scalars $\chi_{i}$ acquire vacuum expectation values (vev) $v_{i}$ and $u$ respectively, breaking the $A_{4}$ symmetry at higher energies, and resulting in the charged lepton mass matrix given as,

$$
M_{e E} M_{e E}^{\dagger}=\left(\begin{array}{cc}
\left(f_{e} v_{1}\right)^{2} I & \left(f_{e} v_{1}\right) M_{E} I \\
\left(f_{e} v_{1}\right) M_{E} I & U_{\omega} \operatorname{Diag}\left[3\left(h_{i}^{e} u\right)^{2}\right] U_{\omega}^{\dagger}+M_{E}^{2} I
\end{array}\right)
$$

where $f_{e}$ and $h_{i}^{e}$ are the Yukawa constants coupling the standard-model fermions to the standard Higgs field and the new scalars respectively. Here $I$ is a $3 \times 3$ unity matrix and $U_{\omega}$ is the magic matrix,

$$
U_{\omega}=\left(\begin{array}{ccc}
1 & 1 & 1 \\
1 & \omega & \omega^{2} \\
1 & \omega^{2} & \omega
\end{array}\right)
$$

with $\omega=e^{2 i \pi / 3}$ and we assume $v_{i} \ll u \ll M_{E}$. With such hierarchy we have a "universal" see-saw scheme for generating the standard-model charged and neutral lepton masses, that translates into a zero-th order neutrino mixing matrix,

$$
U_{v}(\theta)=\left(\begin{array}{ccc}
\cos \theta & -\sin \theta & 0 \\
\sin \theta / \sqrt{2} & \cos \theta / \sqrt{2} & -1 / \sqrt{2} \\
\sin \theta / \sqrt{2} & \cos \theta / \sqrt{2} & 1 / \sqrt{2}
\end{array}\right)
$$

With the discovery of nonzero $\theta_{13}$ by Daya Bay such simple form is now excluded by experimental data, as it leads to zero reactor mixing angle due to a remnant symmetry of $A_{4}$. 

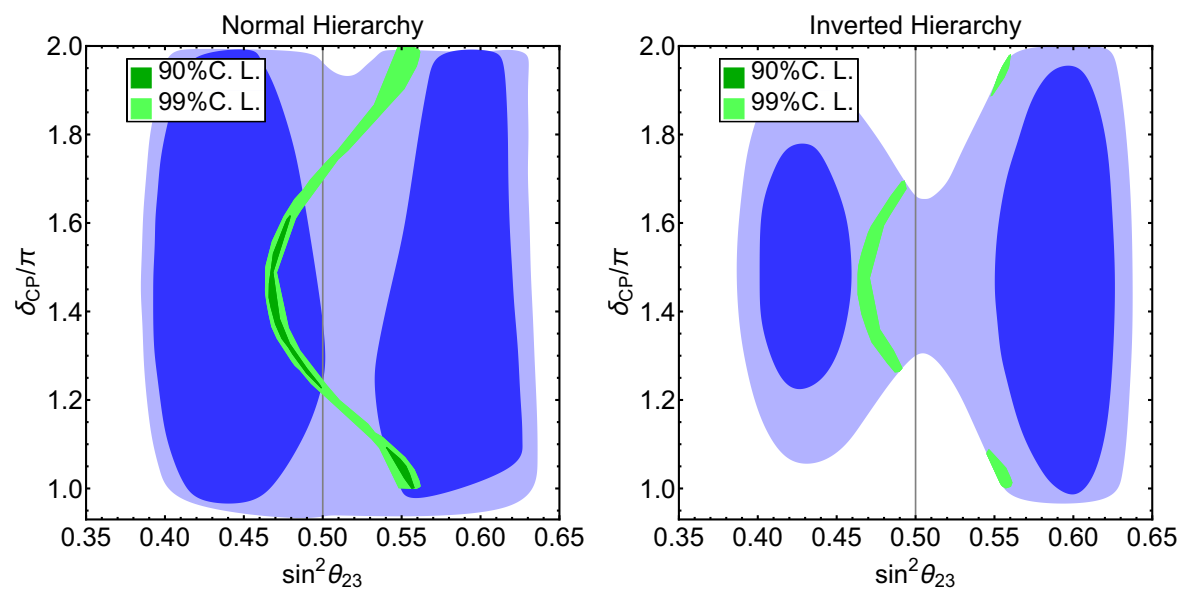

Figure 1: Regions of three-neutrino oscillation paramaters allowed at $90 \%$ and $99 \%$ of C. L. in the unconstrained global fit [1] (dark and light blue, respectively) and within the BMV scenario (dark and light green respectively). The left and right panels correspond to normal and inverted mass ordering, respectively. Figure was taken from [12].

In this letter we focus on the generalized version of the model proposed in [8], by adding to it a single flavon scalar $\xi$ that breaks this remnant $\mu-\tau$ symmetry present in the original version of the model [9], and slightly changes the charged fermion mass matrix to,

$$
M_{e E} M_{e E}^{\dagger}=\left(\begin{array}{cc}
\left(f_{e} v_{1}\right)^{2} I & \left(f_{e} v_{1}\right) Y_{D}^{\dagger} \\
\left(f_{e} v_{1}\right) Y_{D} & U_{\omega} \operatorname{Diag}\left[3\left(h_{i}^{e} u\right)^{2}\right] U_{\omega}^{\dagger}+Y_{D} Y_{D}^{\dagger}
\end{array}\right)
$$

where $Y_{D}=M_{E}\left(I+\beta \operatorname{Diag}\left[1, \omega, \omega^{2}\right]\right)$, and $\beta$ is a small complex parameter. This equation modifies the neutrino mixing matrix to,

$$
U_{v}(\theta) \rightarrow K(\theta, \beta)=U_{\delta}^{\dagger}(\beta) U_{v}(\theta)
$$

where $U_{\delta}^{\dagger}(\beta)$ characterizes the revamping and generates a nonzero reactor mixing angle. Within this revamped scenario $|\beta|$ correlates linearly with $\theta_{13}$ and the phase of $\beta$ induces CP violation in oscillations. In addition, the model also predicts a correlation between the atmospheric mixing angle $\theta_{23}$ and the CP phase. In Fig. 1, we illustrate this predicted correlation between $\theta_{23}$ and $\delta_{C P}$ by numerically varying the model parameters ${ }^{1}$.

we find that, taking into account the most recent global fit of neutrino oscillation paramaters [1], the inverted mass ordering is only allowed at the $99 \%$ of C. L., an enhanced rejection than in the general unconstrained scenario.

On the other hand, the strongly preferred normal ordering case has two solutions, one in each octant of $\theta_{23}$. The preferred solution lies in the lower octant close to maximal $\mathrm{CP}$ violation. The solution for higher octant (close to CP conservation), is still allowed at $90 \%$ of C. L., as seen by the dark green region.

\footnotetext{
${ }^{1}$ For the details of simulation, please refer to [12].
} 


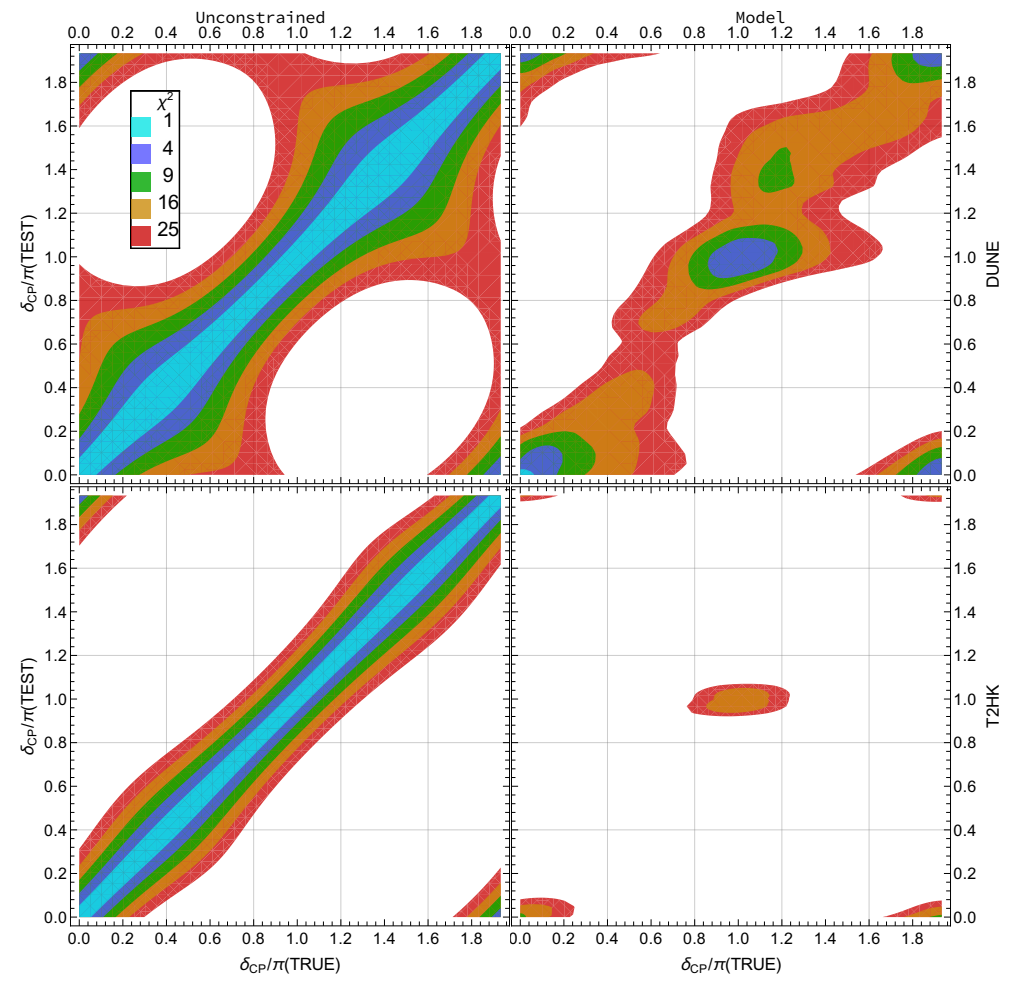

Figure 2: A comparison of the ability to reconstruct the CP phase with and without the inclusion of the revamped BMV model, in the context of DUNE (top row) and T2HK (bottom row).

\section{Results}

We now study how the BMV model can be probed / tested by future long baseline experiments such as DUNE and T2HK.

From Fig. 2, we note that in the standard scenario (left panels), T2HK can reconstruct the values of the $\mathrm{CP}$ phase more faithfully (upto about $5 \sigma$ : - bottom left panel) compared to DUNE (which can reconstruct upto about $3 \sigma$ : - top left panel). From the right panels, we see that the inclusion of the model severely affects the ability to reconstruct the phase.

In Fig. 3, we illustrate how the value of $\theta_{23}$ can be reconstructed upto $5 \sigma$ c.f. in the presence of the BMV model. We note that the recent indication of maximal CP violation $\left(\delta_{C P}=-\pi / 2\right)$ implies that the minima lies in the lower octant ( $1 \sigma$ region i.e. cyan patch in the right panels). Again for CP conservation, the model points to a higher octant (the cyan patch in the left panels). These observations are also consistent with that from Fig. 1.

Now we ask the interesting question of whether one can exhibit the rejection power of future experiments independently of any arbitrarily given choice for the parameters $\theta_{23}$ and $\delta_{C P}$ eventually chosen by nature. Fig. 4 answers this question in the context of DUNE (left panel) and T2HK (right panel), giving quantitative model-testing criteria valid irrespective of any assumed global neutrino oscillation fits. one sees that DUNE can exclude, at $4 \sigma$ statistical significance, the regions corresponding to $\sin ^{2} \theta_{23} \gtrsim 0.59$ and $\sin ^{2} \theta_{23} \lesssim 0.44$ without significant dependence on the value of $\delta_{C P}$ (TRUE). On the other hand, thanks to its higher statistics, T2HK has better sensitivity 


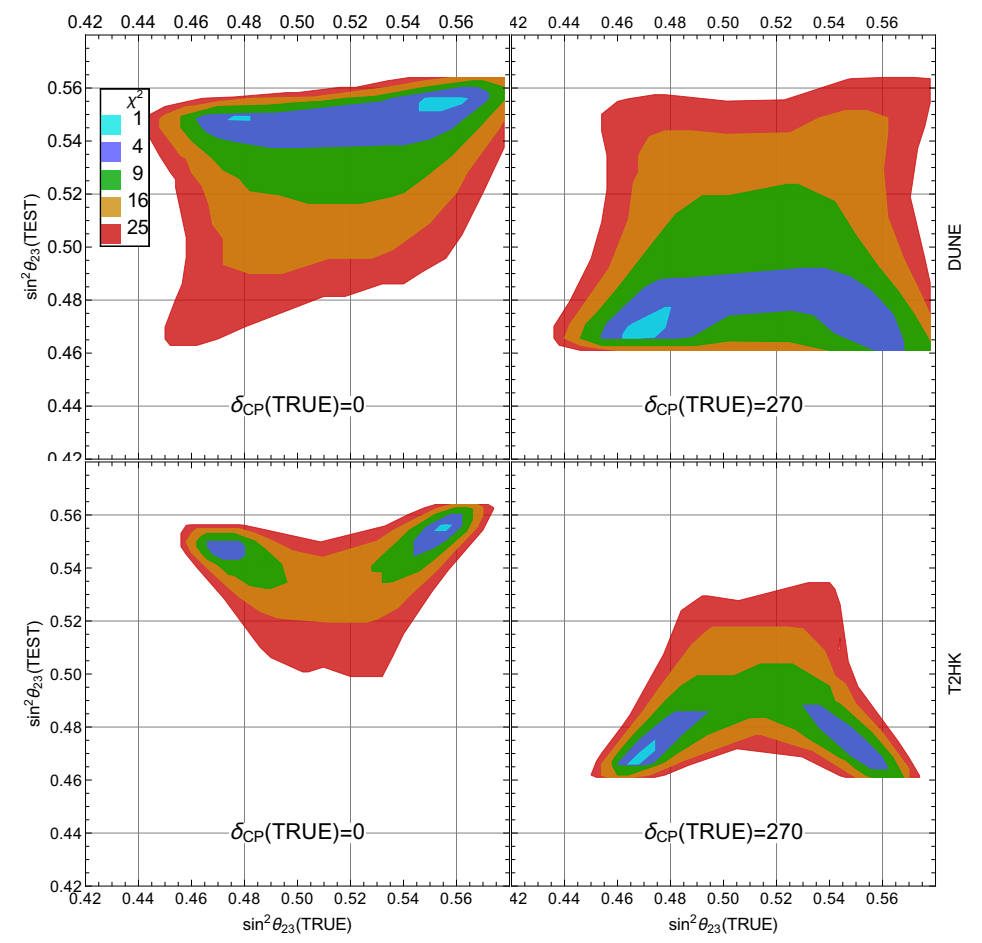

Figure 3: A comparison of the ability to reconstruct the atmospheric angle by DUNE and T2HK with the inclusion of the revamped BMV model, in presence of $\mathrm{CP}$ conservation and maximal $\mathrm{CP}$ violation.

than DUNE and consequently can exclude even larger regions of parameter space. Notice that, as indicated in both panels, the best fit point obtained in [1] lies outside the corresponding $4 \sigma$ sensitivity regions at DUNE and T2HK, indicating how severely such parameter choice would be rejected by these experiments.

\section{Summary and conclusion}

Taking advantage of the latest global determination of neutrino oscillation parameters given in [1] we have investigated the status of the simplest revamped version of the BMV model for neutrino oscillation, proposed in [8], as well as the chances of testing it further at future long-baseline neutrino experiments. By focussing on the sharp correlation between the "poorly determined" oscillation parameters $\theta_{23}$ and the phase $\delta_{C P}$ predicted in the model, we have determined the region of these oscillation parameters allowed within the BMV model, and compared it with what holds in the general three-neutrino oscillation scenario.

We have observed a higher degree of rejection against the higher octant of $\theta_{23}$ than in the general unconstrained case. We have also presented the ability of DUNE and T2HK to probe the model within a robust global approach valid for whatever the choice of $\theta_{23}$ and $\delta_{C P}$ is finally chosen by nature.

\section{Acknowledgments}

M.M. would like to thank the organisers of NuFact 2017 for providing an opportunity of pre- 


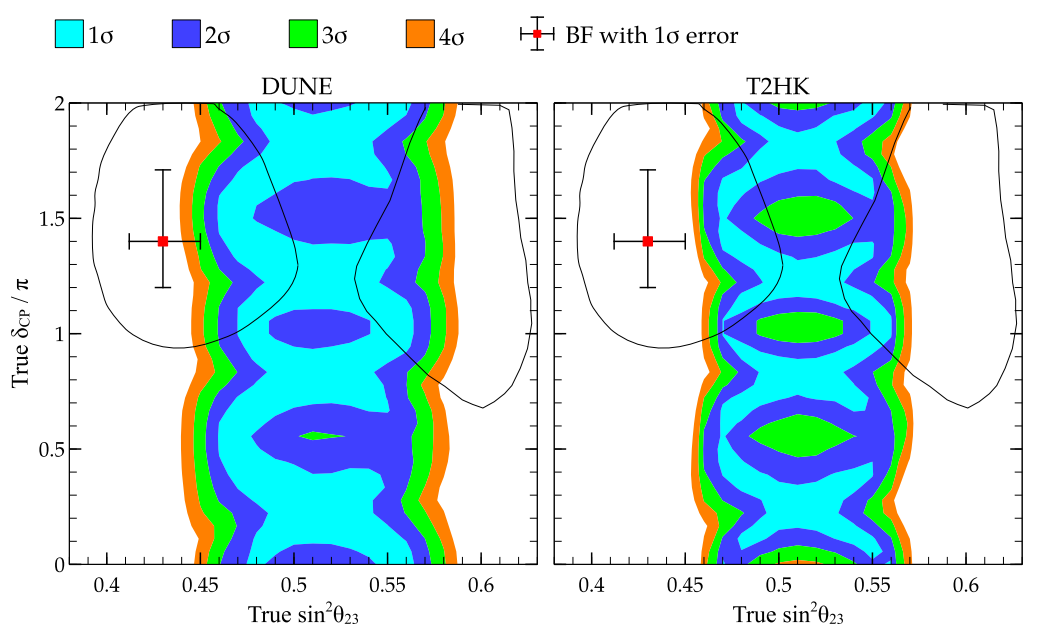

Figure 4: Expected sensitivity regions at various confidence levels at which DUNE (left) or T2HK (right) would test the revamped BMV model. The regions within the black bordered contours correspond to $90 \%$ C.L. and the red square is the current best fit value [1]. The full parameter scan of true values of $\sin ^{2} \theta_{23}$ and $\delta_{C P}$ assumes normal neutrino mass ordering.

senting this work. This work was supported by Spanish grants FPA2014-58183-P, SEV-2014-0398 (MINECO) and PROMETEOII/2014/084 and GV2016-142 (Generalitat Valenciana). P. P. was supported by FAPESP grants 2014/05133-1, 2015/16809-9, 2014/19164-6 and FAEPEX grant N. 2391/17.

\section{References}

[1] P. F. de Salas, D. V. Forero, C. A. Ternes, M. Tortola and J. W. F. Valle, Status of neutrino oscillations 2017, 1708.01186.

[2] Particle Data Group collaboration, C. Patrignani et al., Review of Particle Physics, Chin. Phys. C40 (2016) 100001.

[3] S. Boucenna, S. Morisi, M. Tortola and J. W. F. Valle, Bi-large neutrino mixing and the Cabibbo angle, Phys.Rev. D86 (2012) 051301, [1206.2555].

[4] S. Roy, S. Morisi, N. N. Singh and J. W. F. Valle, The Cabibbo angle as a universal seed for quark and lepton mixings, Phys. Lett. B748 (2015) 1-4, [1410 . 3658].

[5] M. Hirsch, D. Meloni et al., Proceedings of the first workshop on Flavor Symmetries and consequences in Accelerators and Cosmology (FLASY2011), 1201.5525.

[6] S. Morisi and J. W. F. Valle, Neutrino masses and mixing: a flavour symmetry roadmap, Fortsch.Phys. 61 (2013) 466-492, [1206.6678].

[7] H. Ishimori, T. Kobayashi, H. Ohki, H. Okada, Y. Shimizu and M. Tanimoto, An Introduction to Non-Abelian Discrete Symmetries for Particle Physicists. Lecture Notes in Physics. Springer, 2012.

[8] S. Morisi, D. Forero, J. C. Romao and J. W. F. Valle, Neutrino mixing with revamped A4 flavour symmetry, Phys.Rev. D88 (2013) 016003, [1305.6774].

[9] K. S. Babu, E. Ma and J. W. F. Valle, Underlying A(4) symmetry for the neutrino mass matrix and the quark mixing matrix, Phys. Lett. B552 (2003) 207-213, [hep-ph/ 0206292$].$ 
[10] DUNE collaboration, R. Acciarri et al., Long-Baseline Neutrino Facility (LBNF) and Deep Underground Neutrino Experiment (DUNE), 1512.06148.

[11] Hyper-Kamiokande Proto-Collaboration collaboration, K. Abe et al., Physics potential of a long-baseline neutrino oscillation experiment using a J-PARC neutrino beam and Hyper-Kamiokande, PTEP 2015 (2015) 053C02, [1502 . 05199].

[12] S. S. Chatterjee, M. Masud, P. Pasquini and J. W. F. Valle, Cornering the revamped BMV model with neutrino oscillation data, Phys. Lett. B774 (2017) 179-182, [1708.03290]. 